\title{
THE INFLUENCE OF APPLICATION A SIMPLIFIED TRANSFORMATION MODEL BETWEEN REFERENCE FRAMES ECEF AND ECI ONTO PREDICTION ACCURACY OF POSITION AND VELOCITY OF GLONASS SATELLITES
}

\author{
Robert Krzyżek, Bogdan Skorupa \\ AGH University of Science and Technology \\ Faculty of Mining Surveying and Environmental Engineering \\ Department of Geomatics \\ Krakow, Poland
}

\begin{abstract}
In computational tasks of satellite geodesy there is a need for transformation of coordinates between reference frames ECEF - Earth Centered, Earth Fixed and ECI - Earth Centered, Inertial. Strict and simplified transformation models, which can be used in case of the position and velocity short-term predictions of GLONASS satellites, have been presented in this study. Comparison of the results of state vector components predictions of the GLONASS satellites, in dependence of the used transformation model, have also been presented. Accuracy of the prediction has been determined on the basis of the analyse of deviations of the predicted positions and velocities of GLONASS satellites from their values given in broadcast ephemeris.
\end{abstract}

Keywords: broadcast ephemeris, transformation, ECEF, ECI, GLONASS

\section{Introduction}

The GLONASS broadcast ephemeris contain information on position and velocity of satellites in form of Cartesian coordinates and their time derivatives, as well as in form of values of luni-solar accelerations. These data are provided in 30 minute intervals, in global, geocentric reference frame PZ-90.02 (GLONASS Interface Control Document [GLONASS ICD], 2008). This frame, as directly related with the solid Earth, is classified into group of reference frames named as ECEF - Earth Centered, Earth Fixed (Seeber, 2003, Vallado, 2007). Tasks of determination of point position on the Earth surface are realized within such reference frames. Whereas, motion of artificial satellites take place according to the Newton's Mechanics Laws, but this laws are valid only in case of inertial reference frames. Thus in the field of 
computational problems of satellite geodesy, there is need of determination of the satellite position and velocity also in quasi-inertial frame, which is named in literature as ECl - Earth Centered Inertial (Vallado, 2007, Xu, 2008). In (GLONASS ICD, 2008), integration of the equations of satellite motion in $\mathrm{ECl}$ frame is recommended, although usage of simplified algorithm realized in ECEF is permitted. Simplified approach is acceptable due to short prediction interval ( $\pm 15 \mathrm{~min}$ ). In case of longterm satellite orbit prediction or in case of application of an analytical prediction model, calculations in ECl frame should be executed (Góral \& Skorupa, 2012, Hsu et al., 2010).

\section{The Strict and simplified model of transformation the satellite's state vector between frames ECEF and ECI}

According to the Resolution B1.7 XXIV of IAU the General Assembly (Manchester 2000), strict form of transformation of the coordinates between systems ECEF and $\mathrm{ECl}$ is described by the following equation (Kryński, 2004):

$$
\mathbf{X}=\mathbf{Q}(t) \cdot \mathbf{R}(t) \cdot \mathbf{W}(t) \cdot \mathbf{x}
$$

In equation (1) $\mathbf{W}(t)$ is determined as polar motion matrix, $\mathbf{R}(t)$ is considered as Earth rotation matrix, and $\mathbf{Q}(t)$ means precession-nutation matrix, whereas $\mathbf{X}$ and $\mathbf{x}$ are vectors of coordinates determined in $\mathrm{ECl}$ and ECEF respectively. Parameter $t$ is expressed by the equation:

$$
t=\left(T T-2000 \text { January } 01^{d} 12^{h} T T\right) / 36525
$$

In equation (2), TT means Terrestrial Time, expressed in $24 \mathrm{~h}$ periods. A more detailed description of the transformation algorithm described by equation (1) can be found in (Kryński, 2004) and (Xu, 2008).

Equation describing velocity transformation from ECEF into $\mathrm{ECl}$ frame is obtained as result of differentiation of the relation (1), assuming constant matrix $\mathbf{W}(t)$ and $\mathbf{Q}(t)$ (Montenbruck \& Gill, 2000):

$$
\dot{\mathbf{X}}=\mathbf{Q}(t) \cdot \mathbf{R}(t) \cdot \mathbf{W}(t) \cdot \dot{\mathbf{x}}+\mathbf{Q}(t) \cdot \dot{\mathbf{R}}(t) \cdot \mathbf{W}(t) \cdot \mathbf{x}
$$

where

$$
\dot{\mathbf{R}}(t)=\frac{d \theta}{d t} \cdot\left[\begin{array}{ccc}
-\sin \theta & -\cos \theta & 0 \\
\cos \theta & -\sin \theta & 0 \\
0 & 0 & 0
\end{array}\right]
$$

Value of the derivative of Earth rotation angle $\theta$ with respect to time is equal to angular velocity $\omega_{E}$ of the Earth's rotation.

$$
\frac{d \theta}{d t}=\omega_{E}=7.2921151467 \cdot 10^{-5} \frac{\mathrm{rad}}{\mathrm{s}}
$$

Inverse transformation of position and velocity can be realized according to the following relations: 


$$
\begin{gathered}
\mathbf{X}=\mathbf{W}^{\mathrm{T}}(t) \cdot \mathbf{R}^{\mathrm{T}}(t) \cdot \mathbf{Q}^{\mathrm{T}}(t) \cdot \mathbf{X} \\
\dot{\mathbf{X}}=\mathbf{W}^{\mathrm{T}}(t) \cdot \mathbf{R}^{\mathrm{T}}(t) \cdot \mathbf{Q}^{\mathrm{T}}(t) \cdot \dot{\mathbf{X}}+\mathbf{W}^{\mathrm{T}}(t) \cdot \dot{\mathbf{R}}^{\mathrm{T}}(t) \cdot \mathbf{Q}^{\mathrm{T}}(t) \cdot \mathbf{X}
\end{gathered}
$$

Simplified transformation model is also commonly used for solving problems of the prediction of GNSS satellites, assuming that changes of coordinates resulting from the precession-nutation motion and the polar motion are neglected in case of short prediction intervals (GLONASS ICD, 2008, Seeber, 2003). According to the above assumptions, model of coordinate transformation can be described in the following simplified form:

$$
\mathbf{X}=\mathbf{R}(t) \cdot \mathbf{x}
$$

In result of differentiation of the equation (7) we obtain relation describing transformation of the satellite velocity

$$
\dot{\mathbf{X}}=\mathbf{R}(t) \cdot \dot{\mathbf{x}}+\dot{\mathbf{R}}(t) \cdot \mathbf{x}
$$

Approximate inverse transformation of position and velocity can be realized according to the following relations:

$$
\begin{gathered}
\mathbf{x}=\mathbf{R}^{T}(t) \cdot \mathbf{X} \\
\dot{\mathbf{x}}=\mathbf{R}^{\mathrm{T}}(t) \cdot \dot{\mathbf{X}}+\dot{\mathbf{R}}^{\mathrm{T}}(t) \cdot \mathbf{X}
\end{gathered}
$$

Simplified transformation model is convenient for use due to low computational complexity.

\section{Numerical tests}

The numerical tests were aimed at the analysis of the satellite's state vector prediction accuracy, in dependence on used algorithm of transformation from ECEF into $\mathrm{ECl}$ frame. At first, transformations of the position and velocity of the satellite GLN 17, given in broadcast ephemeris from the day 15.01.2015 r., from ECEF into $\mathrm{ECl}$ frame have been made, using simplified algorithm described by the formulas (7) and (8). Then, via prediction, position and velocity of the satellite GLN 17 in successive epochs $t_{\mathrm{k}+1}=t_{k}+60^{\mathrm{m}}$, have been calculated. Coordinate differences $\left(x_{b}-x_{p}, y_{b}-y_{p}, z_{b}-z_{p}\right)$ between predicted position (indicator $p$ ) and position in broadcast ephemeris transformed into $\mathrm{ECl}$ frame (indicator $b$ ) were calculated in the next phase. The differences mentioned above, which were calculated in successive 46 epochs $t_{k+1}$, are shown in Fig. 1. Analogical calculations were made with application of the accurate transformation described by equations (1) - (4). Corresponding deviations of the predicted positions from positions given in broadcast ephemeris were expressed in the $\mathrm{ECl}$ reference frame (Fig. 2). 


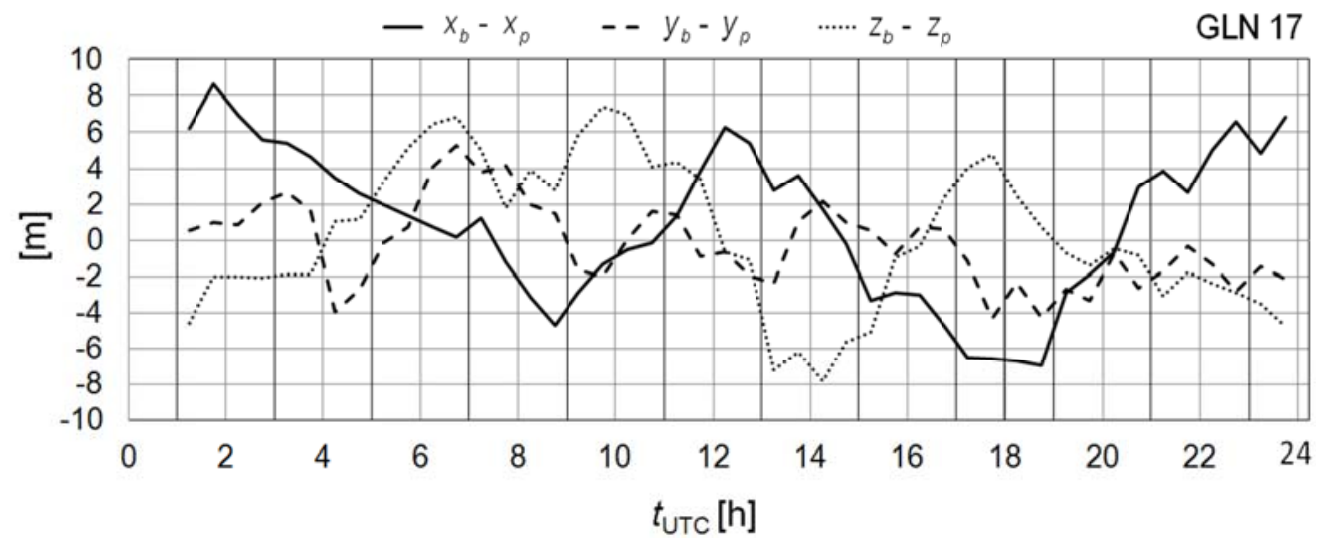

Fig. 1. Differences between coordinates of the satellite GLN 17 given in navigation file and its values predicted forward 60 min., with application of approximate transformation.

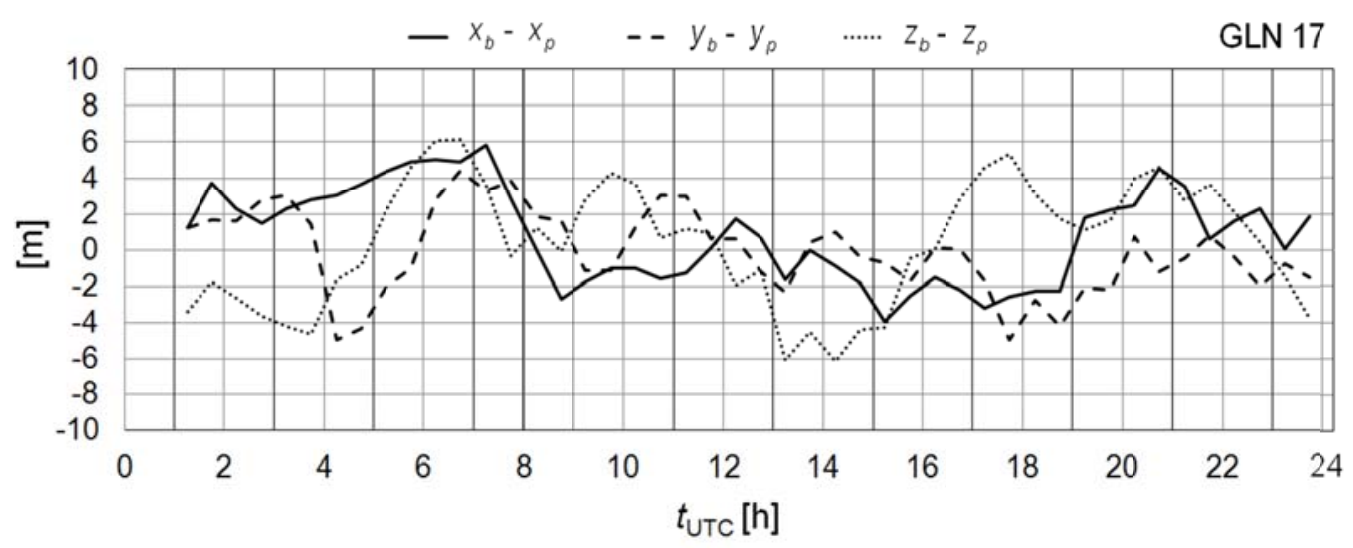

Fig. 2. Differences between coordinates of the satellite GLN 17 given in navigation file and its values predicted forward 60 min., with application of accurate transformation.

Deviations of velocity prediction of the satellite GLN 17 have also been analysed. Differences of velocity components $\left(\dot{x}_{b}-\dot{x}_{p}, \dot{y}_{b}-\dot{y}_{p}, \dot{z}_{b}-\dot{z}_{p}\right)$ are shown in Fig. 3 and Fig.4.

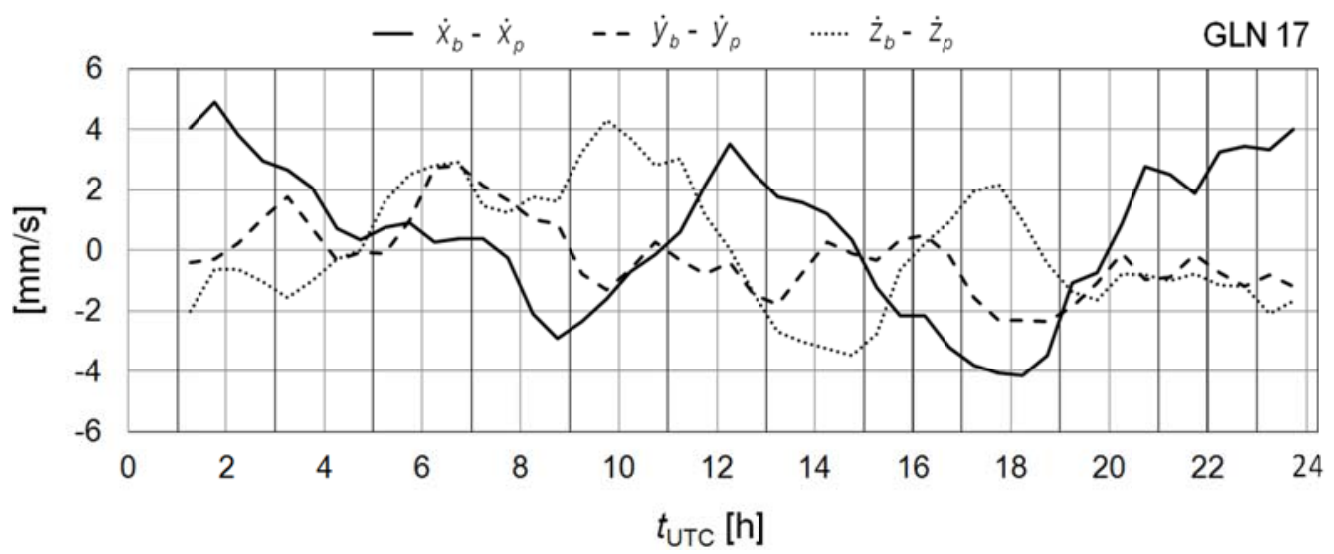

Fig. 3. Differences between velocities of the satellite GLN 17 given in navigation file and its values predicted forward 60 min., with application of approximate transformation. 


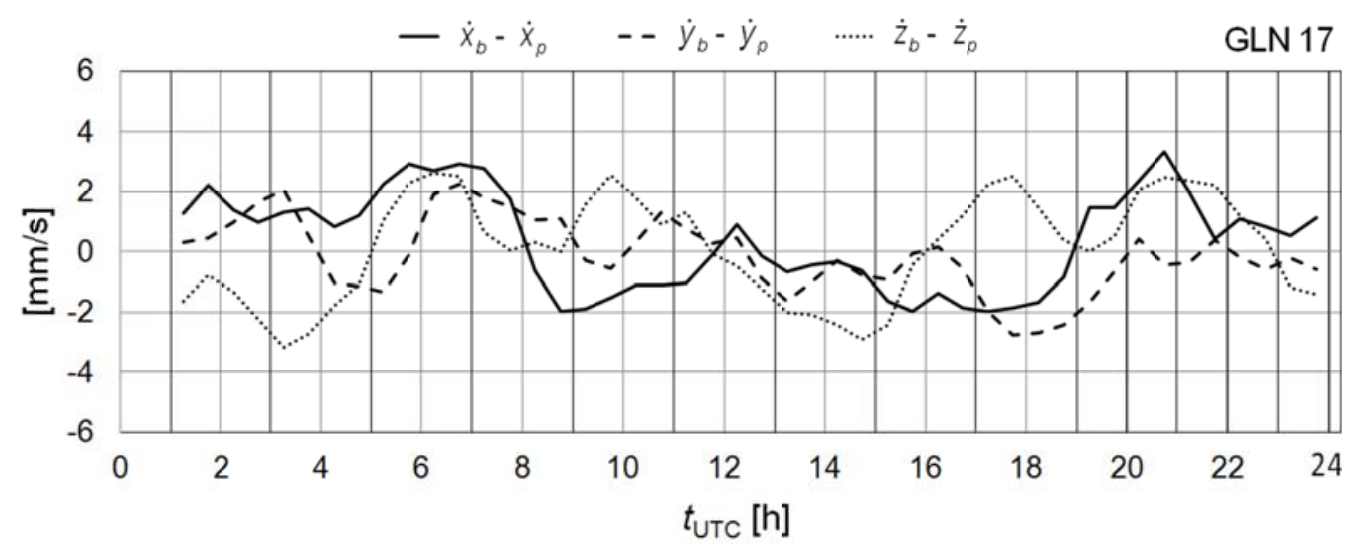

Fig. 4. Differences between velocities of the satellite GLN 17 given in navigation file and its values predicted forward 60 min., with application of accurate transformation.

Deviations of position and velocity obtained in computational process realized with use of accurate transformation model (Fig. 2 and 4), prove considerably smaller amplitudes of daily changes as compared with a variant of calculations made with use of simplified transformation model. However it was observed that character of variability of prediction deviations is to large extend preserved in both computational variants.

In next numerical test, positions and velocities of all 24 GLONASS satellites, in successive epochs $t_{\mathrm{k}+1}=t_{k}+60^{\mathrm{m}}$, were calculated on the basis of data from broadcast ephemeris of the day 15.01.2015. At first, transformations of position and velocity of satellites from ECEF to $\mathrm{ECl}$ frame were executed in two variants: With use of approximate model (variant I) and with use of accurate transformation model (variant II). Comparison of positions and velocities of satellites given in broadcast ephemeris with those calculated on the basis of the prediction were made according to the following equations:

$$
\begin{aligned}
& \delta d=\sqrt{\left(x_{b}-x_{p}\right)^{2}+\left(y_{b}-y_{p}\right)^{2}+\left(z_{b}-z_{p}\right)^{2}} \\
& \delta v=\sqrt{\left(\dot{x}_{b}-\dot{x}_{p}\right)^{2}+\left(\dot{y}_{b}-\dot{y}_{p}\right)^{2}+\left(\dot{z}_{b}-\dot{z}_{p}\right)^{2}}
\end{aligned}
$$

Values $\delta d$ i $\delta v$, calculated for 24 satellites GLONASS, in consecutive 46 epochs $t_{k+1}$, are shown in Fig. 5 and Fig.7 (variant I) and Fig.6 and Fig.8 (variant II). 


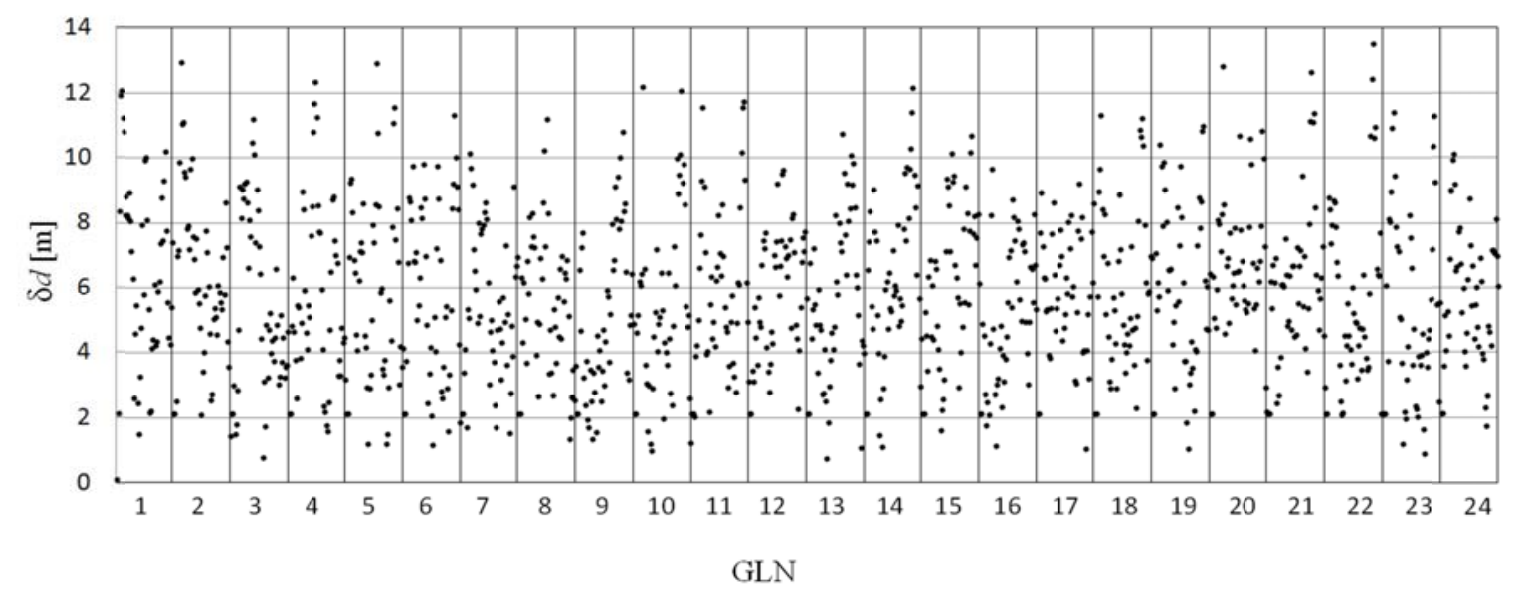

Fig. 5. Distances $\delta d$ between broadcast and predicted satellite positions, obtained in the first variant of calculations, for all GLN satellites (2015-01-15).

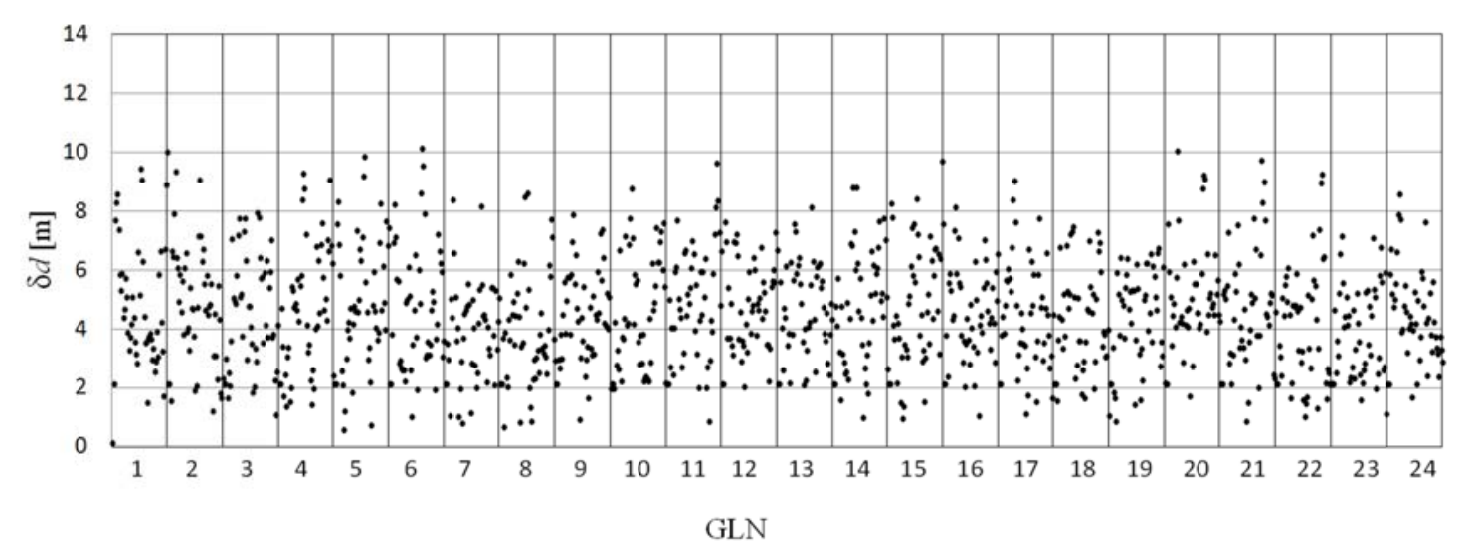

Fig. 6. Distances $\delta d$ between broadcast and predicted satellite positions, obtained in the second variant of calculations, for all GLN satellites (2015-01-15).

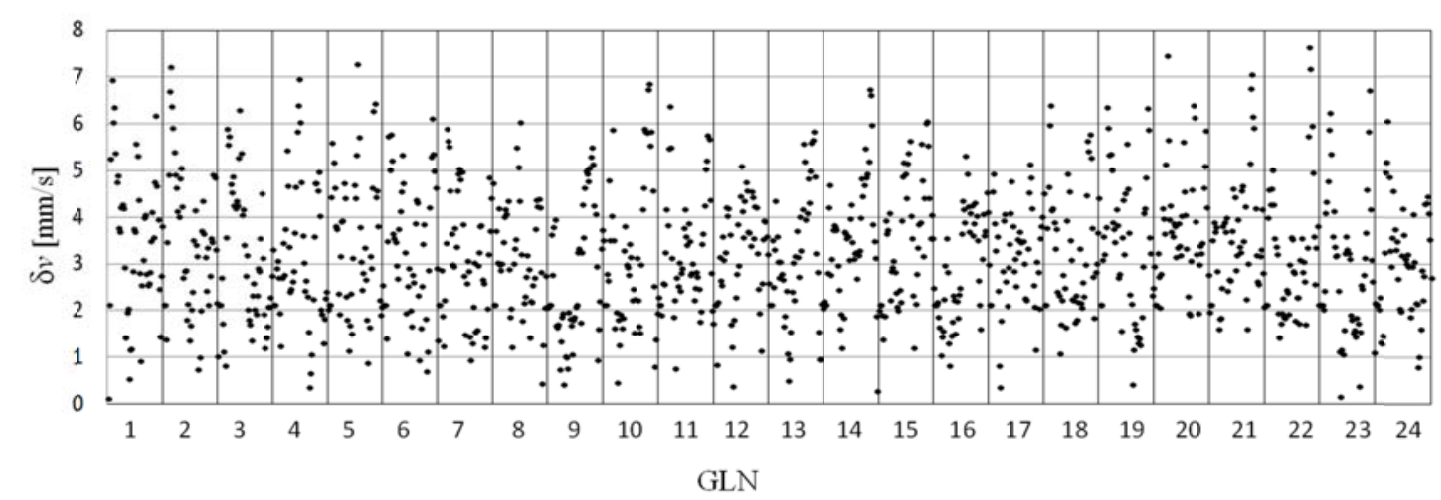

Fig. 7. Differences $\delta v$ between broadcast and predicted satellite velocities, obtained in the first variant of calculations, for all GLN satellites (2015-01-15). 


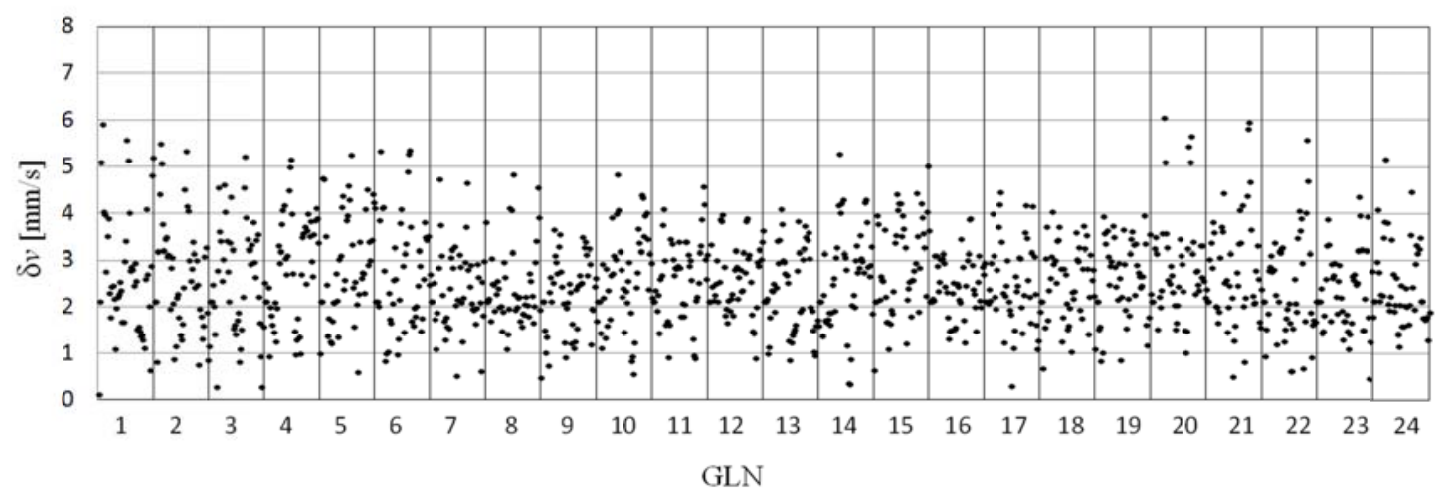

Fig. 8. Differences $\delta v$ between broadcast and predicted satellite velocities, obtained in the second variant of calculations, for all GLN satellites (2015-01-15).

In order to characterize variability of position $\delta d$ and velocity $\delta v$ deviations, their mean values and corresponding standard deviations $\sigma_{\bar{\delta} d}$ and $\sigma_{\bar{\delta}}$, were calculated according to the following relations:

$$
\begin{gathered}
\overline{\delta d}=\frac{\sum_{i=1}^{n} \delta d_{i}}{n}, \quad \overline{\delta v}=\frac{\sum_{i=1}^{n} \delta v_{i}}{n} \\
\sigma_{\delta d}=\sqrt{\frac{\sum_{i=1}^{n}\left(\delta d_{i}-\overline{\delta d}\right)}{n-1}}, \sigma_{\delta v}=\sqrt{\frac{\sum_{i=1}^{n}\left(\delta v_{i}-\overline{\delta v}\right)}{n-1}}
\end{gathered}
$$

where: $n$ - number of compared state vectors, whereas $\delta d_{i}$ and $\delta v_{i}$ - values of position and velocity deviations, calculated on the basis of formulas (11) and (12).

Mean values of state vector deviations and corresponding standard deviations calculated on the basis of relations (13) and (14), realized for described two calculation variants on dependence of prediction interval $\Delta t$, are shown in Table 1.

Table 1. Analysis of values of state vector deviations in dependence on prediction interval $\Delta t$.

\begin{tabular}{|c|c|c|c|c|c|c|}
\hline Calculation variant & \multicolumn{3}{|c|}{$\mathrm{I}$} & \multicolumn{3}{c|}{ II } \\
\hline $\mathrm{D} t[\mathrm{~min}]$ & 30 & 60 & 90 & 30 & 60 & 90 \\
\hline$\overline{\delta d}[\mathrm{~m}]$ & 1.8 & 6.0 & 13.3 & 1.5 & 4.6 & 10.4 \\
\hline$\sigma_{\bar{\delta} d}[\mathrm{~m}]$ & 0.7 & 2.4 & 5.6 & 0.6 & 1.8 & 4.2 \\
\hline$\overline{\delta v}[\mathrm{~mm} / \mathrm{s}]$ & 1.8 & 3.3 & 5.4 & 1.5 & 2.6 & 4.4 \\
\hline$\sigma_{\overline{\delta v}}[\mathrm{~mm} / \mathrm{s}]$ & 0.7 & 1.4 & 2.3 & 0.5 & 1.0 & 1.7 \\
\hline
\end{tabular}


Analytical algorithm based on generalized problem of two fixed centres was applied in calculations of position and velocity of GLONASS satellites (Góral \& Skorupa, 2012). Influence of luni-solar accelerations was taken into consideration according to algorithm given in document GLONASS ICD 2008.

\section{Summary}

Presented in this study simplified transformation algorithm is characterized by low computational complexity. Application of this algorithm for transformation of data given in GLONASS broadcast ephemeris is well justified as short prediction intervals are applied in process of calculation of the GLONASS satellite position and velocity. Application of the accurate algorithm can be needed in case of long prediction intervals or with respect to high precision requirements of transformed data. The numerical tests presented in this study are helpful in assessment of the applicability range of simplified transformation formulas in dependence on precision requirements of the transformed data, as well as on the size of prediction interval of the satellite state vector.

\section{Acknowledgement}

This work has been supported by the project No. 11.11.150.006 at the Department of Geomatics, AGH University of Science and Technology, Krakow, Poland.

\section{References}

GLONASS Interface Control Document (5.1th ed.). (2008). Moscow. Retrieved from http://facility.unavco.org/data/docs/ICD_GLONASS_5.1_(2008)_en.pdf

Góral, W., \& Skorupa, B. (2012). Determination of intermediate orbit and position of GLONASS satellites based on the generalized problem of two fixed centers. Acta Geodynamica et Geomaterialia, 9(3), 283-290

Hsu, T. A., Wang, L. S., Chang, F. R., Tseng, Y. F. (2010). Long-term prediction of GPS satellite orbit. Proceedings of The Society of Instrument and Control Engineers Annual Conference, Taipei, Taiwan, 2906-2909

Kryński, J. (Eds.).(2004). Nowe obowiązujące niebieskie i ziemskie systemy i układy odniesienia oraz ich wzajemne relacje. Warszawa: Instytut Geodezji i Kartografii

Montenbruck, O., Gill, E. (2000). Satellite Orbits - Models, Methods and Applications. Heidelberg: Springer-Verlag

Seeber, G. (2003). Satellite Geodesy: Foundation, Methods \& Applications. Berlin - New York: Walter de Gruyter

Vallado, A. D. (2007). Fundamentals of Astrodynamics and Applications. (3rd ed.) New York: Springer-Verlag 
Xu, G. (2008). Orbits. Berlin: Springer-Verlag

\section{Authors:}

Robert Krzyżek ${ }^{1)}$, rkrzyzek@agh.edu.pl Bogdan Skorupa ${ }^{1)}$, bskorupa@agh.edu.pl

1) AGH University of Science and Technology

Faculty of Mining Surveying and Environmental Engineering

Department of Geomatics, 30 Mickiewicza Av., 30 - 059 Krakow, Poland 